\title{
Impact of preoperative plasma levels of interleukin 6 and interleukin 6 soluble receptor on disease outcomes after radical cystectomy for bladder cancer
}

\author{
Victor M. Schuettfort ${ }^{1,2}\left(\right.$ D $\cdot$ Benjamin Pradere ${ }^{1} \cdot$ Quoc-Dien Trinh $^{3} \cdot$ David D'Andrea $^{1} \cdot$ Fahad Quhal $^{1,4}$. \\ Hadi Mostafaei ${ }^{1,5}$. Ekaterina Laukhtina ${ }^{1,6} \cdot$ Keiichiro Mori ${ }^{1,7} \cdot$ Reza Sari Motlagh $^{1} \cdot$ Michael Rink $^{2}$. \\ Pierre I. Karakiewicz ${ }^{8}$. Piotr Chlosta ${ }^{9}$. Jeremy Yuen-Chun Teoh ${ }^{10}$. Yair Lotan ${ }^{11}$. Douglas Scherr ${ }^{12}$. \\ Mohammad Abufaraj ${ }^{1,13}$. Marco Moschini ${ }^{14,15,16}$. Shahrokh F. Shariat ${ }^{1,6,11,12,13,17,18,19}$
}

Received: 15 February 2021 / Accepted: 26 April 2021 / Published online: 23 May 2021

(c) The Author(s) 2021

\begin{abstract}
Background Preoperative plasma levels of Interleukin 6 (IL6) and its soluble receptor (IL6sR) have previously been associated with oncologic outcomes in urothelial carcinoma of the bladder (UCB); however, external validation in patients treated with radical cystectomy (RC) for UCB is missing.

Patients/methods We prospectively collected preoperative plasma from 1,036 consecutive patients at two institutes. These plasma specimens were assessed for levels of IL6 and IL6sR. Logistic and Cox regression analyses were used to assess the correlation of plasma levels with pathologic and survival outcomes. The additional clinical net benefits of preoperative IL6 and IL6sR were evaluated using decision curve analysis (DCA).

Results Median IL6 and IL6sR plasma levels were significantly higher in patients with adverse pathologic features. Elevated biomarker levels were independently associated with an increased risk for lymph node metastasis and $\geq$ pT3 disease. Both biomarkers were independently associated with recurrence-free survival (RFS), cancer-specific survival (CSS) and overall survival (OS). The addition to, respectively, fitted pre- and postoperative prognostic models improved the predictive accuracy for lymph node metastasis, $\geq$ pT3 disease, RFS and CSS on DCA.

Interpretation We confirmed that elevated preoperative plasma levels of IL6 and IL6sR levels are associated with worse oncological disease survival in patients treated with RC for UCB in a large multicenter study. Both biomarkers hold potential in identifying patients with adverse pathological features that may benefit from intensified/multimodal therapy and warrant inclusion into predictive/prognostic models. They demonstrated the ability to improve the discriminatory power of such models and thus guide clinical decision making.
\end{abstract}

Keywords MIBC · Biomarker · Bladder cancer · Interleukin 6 - Interleukin 6 soluble receptor · Urothelial carcinoma

\section{Introduction}

Radical cystectomy (RC) is the standard of care for very high risk and Bacillus Calmette-Guerin (BCG) unresponsive non-muscle invasive bladder cancer (NIMBC) as well as muscle invasive bladder cancer (MIBC) [1-4]. Despite adequate therapy with curative intent, a significant proportion of patients are misstaged, resulting in suboptimal outcomes

Shahrokh F. Shariat

shahrokh.shariat@meduniwien.ac.at

Extended author information available on the last page of the article
[5-9]. Patient selection for tailored therapy remains challenging, as we are still lacking clinically reliable biomarkers for outcome prediction [9-11]. So far, the best prognostic models are based on postoperative pathologic features, while preoperative outcome prediction remains inaccurate, although it is, at least, of equal importance [11-15]. Novel biomarkers that are readily available and sufficiently improve current predictive and prognostic models for outcome prediction are desperately needed, in order to accurately predict the clinical behavior of each tumor in each patient at that time $[10,11,16,17]$.

As pro-inflammatory cytokines were found to be essential in the pathogenesis of urothelial carcinoma of the bladder 
(UCB), elevated pretreatment plasma levels of these markers might be useful for prediction of outcome [18]. Indeed, experimental data indicate that elevated levels of interleukin 6 (IL-6), a pro-inflammatory pleiotropic cytokine, are associated with aggressive tumor behavior in UCB $[19,20]$. Overexpression of IL6 and its soluble receptor (IL6sR) has been reported in several malignancies, including prostate cancer and UCB [21-23]. In UCB, Andrews et al. reported an association of preoperative blood levels of IL6 and IL6sR on oncological survival outcomes in UCB, but their study was limited by their single-center nature, small sample sizes and lack of advanced statistical analyses [17, 23].

To externally validate the relationship of preoperative plasma IL-6 and IL-6sR levels with established features of bladder cancer invasion, metastasis and survival outcomes, we studied a large consecutive cohort of patients with nonmetastatic advanced UCB treated with RC and pelvic lymphadenectomy. We hypothesized that patients with non-metastatic advanced UCB harboring occult metastases would have elevated levels of plasma IL-6 and IL-6sR which would be associated with features of biologically and clinically aggressive disease as well as poor survival despite effective local disease control. Our aim was to identify patients who are most likely to benefit from an intensified perioperative systemic therapy [24]. Beyond multivariable modeling, we used predictive accuracy testing and decision curve analysis (DCA) to assess real-world clinical utility of preoperative blood levels of IL6 and IL6sR as biomarkers.

\section{Methods}

\section{Patients selection}

All procedures described in the present study were undertaken with the approval and oversight of the Institutional Review Board for the Protection of Human Subjects. This study is retrospective analysis of prospectively collected consecutive cohort of patients who were treated with RC for non-metastatic UCB at two medical institutions. The extent of lymphadenectomy and choice of urinary diversion were at the surgeon's discretion. Patients with any concomitant secondary malignancy, concomitant upper urinary tract carcinoma or missing data were excluded. No patient received neoadjuvant chemotherapy or radiotherapy. Adjuvant chemotherapy was administered to 167 patients $(16.1 \%)$ at the clinicians' discretion based on tumor stage and overall health status. No patient received adjuvant radiotherapy.

\section{Biomarker measurements}

Preoperative serum and plasma samples were collected typically on the morning of the day of surgery after an overnight fast. Specimen collection and measurement have been described in detail previously [25]. Briefly, blood was collected into Vacutainer CPT 8-ml tubes containing $0.1 \mathrm{ml}$ of $1 \mathrm{~m}$ sodium citrate (Becton Dickinson, Franklin Lakes, NJ) and centrifuged at room temperature for $20 \mathrm{~min}$ at $1500 \times \mathrm{g}$. The top layer corresponding to plasma was decanted using sterile transfer pipettes and immediately frozen and stored at $-80{ }^{\circ} \mathrm{C}$ in polypropylene cryopreservation vials (NalgeNunc, Rochester, NY). For quantitative measurements of IL-6 and IL-6sR levels, we used quantitative immunoassays (R\&D Systems, Minneapolis, MN). Every sample was run in duplicate, and the mean was used. Differences between the two measurements for IL-6 and IL-6sR were minimal (intra-assay precision coefficients of variation: $5.2 \pm 3.1$, and $3.6 \pm 2.9 \%$, respectively).

\section{Pathological review and follow-up}

All surgical specimens were processed according to standard pathological procedures as previously described [7]. All cases were histologically confirmed urothelial carcinoma of the bladder with only minor secondary variant components, if any. Genitourinary pathologists assigned tumor grade according to the 1973 WHO grading system. Pathologic stage was reassigned according to the 2002 American Joint Committee on Cancer TNM staging system. The presence of concomitant carcinoma in situ (CIS) was defined as the presence of CIS in conjunction with another tumor other than CIS [26]. Pelvic lymph nodes were examined grossly, and all lymphoid tissue was submitted for histological examination. Positive soft tissue surgical margin was defined as the presence of tumor at inked areas of soft tissue on the RC specimen [5]. Urethral or ureteral margins were not considered as soft tissue surgical margins. Lymphovascular invasion was defined as the unequivocal presence of tumor cells within an endothelium-lined space without underlying muscular walls [27].

Clinical and radiological follow-up was performed in accordance with institutional protocols and current guidelines. Routine follow-up usually included physical examination, radiological imaging and urine cytology every three months for two years. Between the second and the fifth year, follow-up was performed semiannually. Afterwards, in most cases, an annual follow-up was performed. Tumor recurrence was defined as the occurrence of locoregional recurrence or distant metastasis on radiological imaging. Cause of death was abstracted from medical charts and/or from death certificates [28].

\section{Statistical analysis}

Report of categorical variables included frequencies and proportions. Reporting of continuous coded variables 
focused on medians and interquartile ranges (IQR). With respect to preoperative plasma levels of IL6 and IL6sR, which were treated as continuous variables, group comparisons were performed using the Mann-Whitney U tests, Kruskal-Wallis tests or calculation of Spearman's rank correlation coefficient $(r)$ and subsequent significance testing, as appropriate. For analyses that required group classification, such as Kaplan-Meier survival curves or 5-year survival rates, stratification (low vs. high) was performed using median plasma levels of IL6 and IL6sR.

Binominal logistic regression analysis was performed for evaluating the association of preoperative plasma levels of IL6 and IL6sR with lymph node metastasis, $\geq$ pT3 disease or any non-organ confined disease (NOCD, defined as $\geq$ pT3 disease and/or lymph node metastasis). The area under the curve (AUC) of receiver operating characteristics (ROC) curves was calculated to determine the predictive accuracy of multiple logistic regression models. DeLong's test was used to test for statistical significance between different AUCs. Association between preoperative IL6 and IL6sR with recurrence-free survival (RFS), cancer-specific survival (CSS) and overall survival (OS) was assessed in univariable and multivariable Cox regression models. Clinical and pathological tumor grade was excluded as a variable for all predictive models, since virtually all RC patients had high-grade UCB. Separate Cox regression models that featured either preoperative clinical variables or postoperative histopathological variables were created. The discriminative ability of these models after inclusion of IL6 and/or IL6sR was tested using Harrel's concordance index (C-index). The additional clinical net benefit of both markers was also evaluated using decision curve analysis (DCA)[29]. Again, separate reference models that represented either the preor postoperative setting were created, to which IL6 and/or IL6sR were included in order to assess the additional predictive value of each biomarker. All reported p-values were two-sided, and statistical significance was set at 0.05. All statistical analyses were performed using $\mathrm{R}$ version 3.6.3.

\section{Results}

\section{Association with clinicopathologic features}

A total of 1,036 patients were included in the analysis. The median age of the entire cohort was 66.5 years (IQR 59.7-72.7). Median plasma levels of IL6 and IL6sR were significantly higher among patients with adverse pathologic features such as lymphovascular invasion, lymph node metastasis and advanced pathologic tumor stage ( $p$-values $<0.05$, Table 1$)$. There was no relevant correlation between age and IL6 or IL6sR plasma levels $(r=0.05$, $p=0.08$ and $r=0.1, p<0.001$, respectively). There was also no correlation between plasma levels of both biomarkers $(r=0.05, p=0.13$ ) or between the number of lymph nodes removed and either IL6 $(r=0.005, p=0.86)$ or IL6sR $(r=0.11, p=0.57)$.

On multivariable logistic regression modeling, elevated preoperative plasma levels of both IL6 and IL6sR were significantly associated with an increased risk of $\geq \mathrm{pT} 3$ disease, lymph node metastasis and any NOCD ( $p$-values $<0.03$, Table 2). ROC curve analysis showed that the addition of preoperative plasma levels of IL6 and IL6sR to a reference model comprising age, sex and clinical tumor stage significantly improved the discriminating ability for prediction of lymph node metastasis $(5 \%, p<0.001), \geq$ pT3 disease $(4 \%$, $p<0.001)$ and any NOCD $(4 \%, p<0.001$, Table 2$)$. There were no significant differences between preoperative plasma levels of IL6 and IL6sR with respect to change of AUC. The highest values were achieved through addition of both markers. On DCA for prediction of $\geq$ pT3 disease, lymph node metastasis and any NOCD, only the addition of pretreatment plasma levels of IL6 to the previously described reference model resulted in a slight improvement of the clinical net benefit for prediction of lymph node metastasis, $\geq$ pT3 disease and any NOCD (Fig. 1).

\section{Association of survival outcomes with preoperative clinical variables}

Median follow-up of patients alive was 37 months (IQR 14.5-108.5). The 5-year estimates for RFS, CSS and OS were $62.5 \%$ (95\% CI 59.2-66\%), 66\% (95\% CI $63.3-70 \%$ ) and $57 \%$ (95\% CI 53.6-60.5\%), respectively. Patients who experienced disease recurrence or died of UCB had significantly higher median pretreatment plasma levels of IL6 and IL6sR $(p<0.001)$. Patients who died of any cause also had significant higher median pretreatment plasma levels of IL6sR $(p<0.001)$, but not IL6 $(p=0.29)$. When stratified by median IL6 and IL6SR levels, patients with elevated plasma levels had significant worse survival outcomes with respect to RFS, CSS and OS (Fig. 2).

In a multivariable Cox regression model that included established preoperative available variables (age, sex and clinical tumor stage), higher pretreatment plasma levels of both IL6 and IL6sR were independently associated with worse RFS, CSS and OS (Table 3). The addition of preoperative plasma levels of both IL6 and IL6sR improved the $\mathrm{C}$-indices of the same model for early prediction of RFS (11.4\%), CSS (10.1\%) and OS (4.4\%). On DCA, only the addition of pretreatment IL6sR plasma levels to the same Cox model slightly improved the clinical net benefit of the model for early prediction of RFS and CSS between a threshold probability of $10-40 \%$. There was no improvement for the prediction of OS across any threshold probability (Fig. 1). 
Table 1 Association of median preoperative plasma levels of il6 and il6sr with respect to clinicopathologic characteristics in 1036 patients treated with radical cystectomy for urothelial carcinoma of the bladder

\begin{tabular}{|c|c|c|c|c|c|c|}
\hline Variable & & $\mathrm{n}(\%)$ & $\begin{array}{l}\text { median plasma IL6 } \\
\text { (IQR) } \mathrm{pg} / \mathrm{mL}\end{array}$ & $p$ & $\begin{array}{l}\text { median plasma IL6SR } \\
\text { (IQR) } \mathrm{ng} / \mathrm{mL}\end{array}$ & $p$ \\
\hline Overall & & $1036(100)$ & $2.76(2.12-3.99)$ & & $25.83(20.6-30.64)$ & \\
\hline \multirow[t]{2}{*}{ Age (stratified by median age) } & $<66.5$ years & 517 & $2.72(2.11-3.92)$ & 0.25 & $25.4(20.4-30.3)$ & 0.31 \\
\hline & $>66.5$ years & 519 & $2.77(2.13-4.19)$ & & $26.4(20.7-31.1)$ & \\
\hline \multirow[t]{2}{*}{ Sex } & Male & $814(78.6)$ & $2.76(2.13-3.98)$ & 0.89 & $25.85(20.6-30.4)$ & 0.65 \\
\hline & Female & $222(21.4)$ & $2.75(2.06-4.10)$ & & $25.81(20.5-31.38)$ & \\
\hline \multirow[t]{2}{*}{ Blood transfusion } & Yes & $268(25.9)$ & $2.91(2.22-4.67)$ & 0.018 & $26.93(21.28-32.2)$ & 0.02 \\
\hline & No & $768(74.1)$ & $2.69(2.09-3.82)$ & & $25.4(20.3-30.32)$ & \\
\hline \multirow[t]{2}{*}{ Thrombocytosis } & Yes & $113(10.9)$ & $2.77(2.09-4.0)$ & 0.41 & $26.4(20.5-31.23)$ & 0.45 \\
\hline & No & $923(89.1)$ & $2.29(2.71-4.0)$ & & $25.8(20.6-30.45)$ & \\
\hline \multirow[t]{2}{*}{ Clinical tumor grade } & Grade 2 & $6(0.6)$ & $2.89(2.72-3.14)$ & 0.6 & $21.3(19.0-33.4)$ & 0.61 \\
\hline & Grade 3 & $1022(98.6)$ & $2.74(2.10-3.98)$ & & $25.88(20.6-30.6)$ & \\
\hline \multirow[t]{6}{*}{ Clinical tumor stage } & $\mathrm{cTa}$ & $23(2.2)$ & $2.71(2-3.58)$ & 0.44 & 23.7 (19.9-28.44) & 0.11 \\
\hline & cTis & $105(10.1)$ & $2.93(2.23-4.69)$ & & $23.6(20.2-29.2)$ & \\
\hline & $\mathrm{cT} 1$ & $336(32.4)$ & $2.65(2.03-3.8)$ & & $26.15(20.9-31.1)$ & \\
\hline & cT2 & $498(48.1)$ & $2.77(2.1-4.16)$ & & $26.28(20.6-30.88)$ & \\
\hline & cT3 & $38(3.7)$ & $2.84(2.11-3.43)$ & & $29.2(20.61-32.6)$ & \\
\hline & cT4 & $29(2.8)$ & $2.83(2.33-3.92)$ & & $25.2(21.1-29)$ & \\
\hline \multirow[t]{3}{*}{ Pathological tumor grade } & Grade 1 & $62(6.0)$ & $2.43(2.11-3.01)$ & 0.055 & $23.85(18.02-30.2)$ & 0.011 \\
\hline & Grade 2 & $11(1.1)$ & $2.33(2.13-2.82)$ & & $20.6(19.7-22.4)$ & \\
\hline & Grade 3 & $963(93.0)$ & $2.79(2.13-4.09)$ & & $26(20.7-30.8)$ & \\
\hline \multirow[t]{7}{*}{ Pathological tumor stage } & pT0 & $62(6.0)$ & $2.43(2.12-3.01)$ & $<0.001$ & $23.85(18.02-30.2)$ & $<0.001$ \\
\hline & $\mathrm{pTa}$ & $22(2.1)$ & $2.46(2.32-3.11)$ & & $20.6(19.33-23.58)$ & \\
\hline & pTis & $131(12.6)$ & $2.83(2.21-3.9)$ & & $23.7(20.35-27.9)$ & \\
\hline & pT1 & $162(15.6)$ & $2.47(1.91-3.33)$ & & $26.85(21.73-30.14)$ & \\
\hline & pT2 & 248 (23.9) & $2.67(1.93-3.69)$ & & $25.3(20.3-30.23)$ & \\
\hline & pT3 & $281(27.1)$ & $2.93(2.22-4.85)$ & & $27.7(22.4-32.56)$ & \\
\hline & pT4 & $130(12.5)$ & $3.04(2.33-4.74)$ & & $26.49(20.53-32.58)$ & \\
\hline \multirow[t]{2}{*}{ Upstaged to $\geq$ pT 3 disease } & Yes & $357(34.7)$ & $2.98(2.23-4.87)$ & $<0.001$ & $27.6(22.2-32.7)$ & $<0.001$ \\
\hline & No & $672(65.3)$ & $2.65(2.02-3.56)$ & & $25(20.3-29.8)$ & \\
\hline \multirow[t]{2}{*}{ Soft tissue surgical margin } & Positive & $95(9.2)$ & $2.83(2.26-4.43)$ & 0.09 & $28.3(24.25-34.85)$ & 0.001 \\
\hline & Negative & $941(90.8)$ & $2.73(2.11-3.97)$ & & $25.5(20.4-30.37)$ & \\
\hline \multirow[t]{2}{*}{ Lymphovascular invasion } & Positive & $295(28.5)$ & $2.96(2.28-4.74)$ & $<0.001$ & $26.4(21.4-31.8)$ & 0.042 \\
\hline & Negative & $741(71.5)$ & $2.65(2.02-3.69)$ & & $25.7(20.4-30.1)$ & \\
\hline \multirow[t]{2}{*}{ Concomitant Carcinoma in situ } & Yes & $572(55.2)$ & $2.81(2.13-3.97)$ & 0.74 & $25.33(20.4-30.1$ & 0.08 \\
\hline & No & $464(44.8)$ & $2.69(2.12-4.13)$ & & $26.43(20.98-31.25)$ & \\
\hline \multirow[t]{2}{*}{ Lymph node metastasis } & Yes & $263(25.4)$ & $3.21(2.35-4.8)$ & $<0.001$ & $27.57(22.19-32.05)$ & $<0.001$ \\
\hline & No & 773 (74.6) & $2.63(1.95-3.69)$ & & $25.3(20.3-30.1)$ & \\
\hline \multirow[t]{2}{*}{ Use of adjuvant chemotherapy } & Yes & $167(16.1)$ & $2.99(2.27-4.31$ & 0.018 & $28.1(21.84-32.35)$ & 0.005 \\
\hline & No & 869 (83.9) & $2.7(2.09-3.92)$ & & $25.5(20.4-30.2)$ & \\
\hline \multirow[t]{2}{*}{ Recurrence } & Yes & $335(32.3)$ & $3.15(2.32-5.04$ & $<0.001$ & $30.6(25.65-37.3)$ & $<0.001$ \\
\hline & No & $701(67.7)$ & $2.62(1.94-3.59)$ & & $23.7(20.00-28.2)$ & \\
\hline \multirow[t]{2}{*}{ Death of bladder cancer } & Yes & $303(29.2)$ & $3.11(2.32-5.0)$ & $<0.001$ & $31.0(26.35-37.4)$ & $<0.001$ \\
\hline & No & 733 (70.8) & $2.64(1.96-3.66)$ & & $23.7(20.0-28.3)$ & \\
\hline \multirow[t]{2}{*}{ Death of any cause } & Yes & $564(54.4)$ & $2.77(2.22-3.98)$ & 0.29 & $27.90(22.48-34.28)$ & $<0.001$ \\
\hline & No & $472(45.6)$ & $2.73(2.03-3.99)$ & & $23.63(20.0-28.1)$ & \\
\hline
\end{tabular}

Bold mean that result is significant, statistical significance was set at $p<0.05$ 


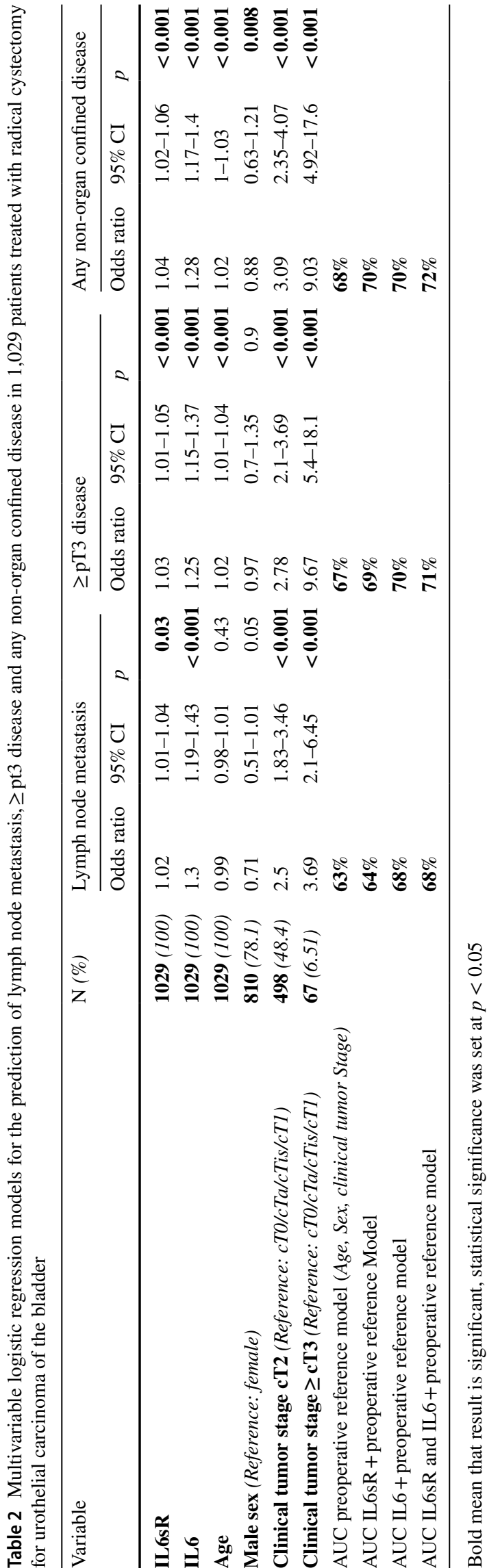

\section{Association of survival outcomes with postoperative histopathological variables}

In a multivariable Cox regression model that focused on established postoperative variables, higher pretreatment plasma levels of both IL6 and IL6sR remained independently associated with worse RFS, CSS and OS (Table 3). The addition of preoperative plasma levels of both IL6 and IL6sR to the same prognostic model slightly improved the C-indices for prediction of RFS (4\%), CSS (3.6\%) and OS (1.5\%). On DCA, only the addition of preoperative plasma levels of IL6sR slightly improved the clinical net benefit of the model for prediction of RFS and CSS between a threshold probability of $20-50 \%$. Again, there was no improvement for the prediction of OS across any threshold probability (Fig. 1).

\section{Discussion}

Despite additional studies that have added to our knowledge about molecular markers in UCB, no biomarker is used for individualized treatment recommendations, except urine cytology [11]. This is largely due to the lack of external validation in large, multicenter studies. In order to conclusively assess the potential predictive value of plasma levels of IL6 and IL6sR, we performed an external validation of its predictive value in an independent, large, multi-institutional cohort of patients who were treated with RC for UCB [17, $30,31]$. We were able to validate and extend our previous findings that elevated preoperative plasma levels of both biomarkers are independent predictors of lymph node metastasis and $\geq \mathrm{pT} 3$ disease on logistic regression analyses [23]. Therefore, both biomarkers demonstrated the potential to identify patients with adverse pathologic features associated with aggressive biological and clinical behavior. These biomarkers, as part of a panel, could help in identifying those patients who may benefit from intensified/multimodal perioperative systemic therapy. Furthermore, we confirmed the independent association of elevated preoperative IL6 and IL6sR with worse survival outcomes.

It is well documented that inflammation is associated with the development and progression of cancer. IL-6 and IL6SR are produced by various normal cells, but also tumor-infiltrating immune cells and tumor cells themselves. It has therefore previously been concluded that increased systemic IL-6 levels are a result of local tumor production. Indeed, Andrews et al. found that plasma levels of IL-6 and IL-6sR are associated with tumor stage and metastases and are strong independent predictors of disease recurrence and disease-specific survival [23]. However, the question of whether one or both of these biomarkers can improve the prognostic use of established predictors of cancer outcome 

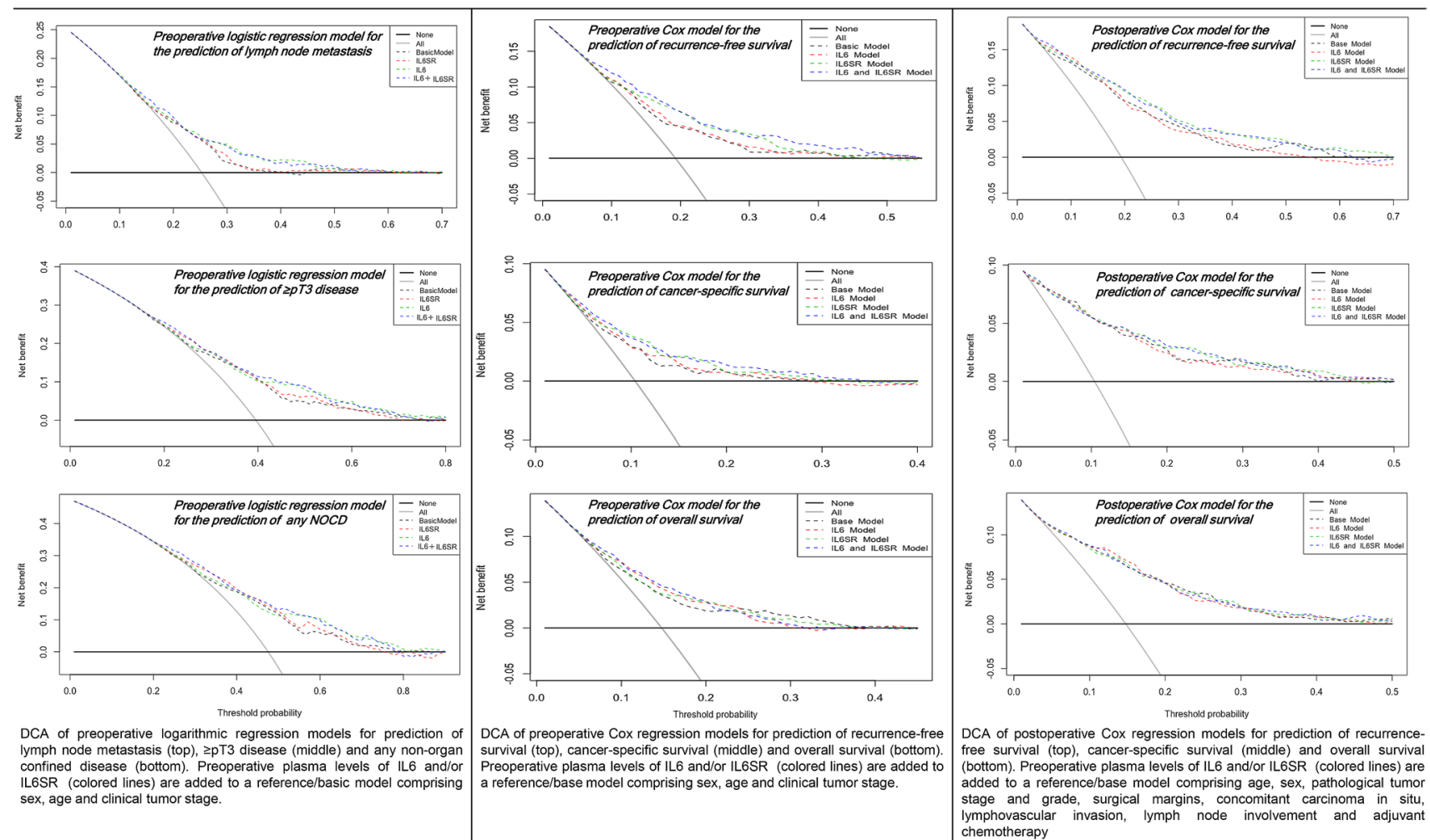

DCA of preoperative logarithmic regression models for prediction of lymph node metastasis (top), $\geq \mathrm{pT} 3$ disease (middle) and any non-organ
confined disease (bottom). Preoperative plasma levels of IL6 and/or IL6SR (colored lines) are added to a reference/basic model comprising sex, age and clinical tumor stage. lymphovascular
chemotherapy

Fig. 1 Decision curve analysis (dca) for the additional net benefit of il6 and il6sr based on separate reference models for the outcome prediction in 1,036 patients treated with radical cystectomy for urothelial carcinoma of the bladder. Description the x-axis is the threshold probabilities. The y-axis measures the net benefit which is calculated by adding the true positives and subtracting the false-positives. The horizontal line representing the $\mathrm{x}$-axis assumes that no patients

requires more than the conventional multivariable analyses in small cohorts, as previously performed by Andrews et al. [23]. It must be established that the use of a prognostic biomarker adds unique information that improves the performance of a predictive model constructed without the new biomarker by a statistically significant margin $[17,30]$. Therefore, we tested whether preoperative plasma IL6 and/ or IL6sR improved the accuracy of predictive models and whether they added net clinical benefit in the pre- and/or postoperative setting [23]. We found that the addition of preoperative IL6 and IL6sR to a reference model, which included only preoperatively available variables, significantly improved the discriminatory power for prediction of non-organ confined disease. Both biomarkers also demonstrated the ability to improve the performance of the same model for early prediction of RFS $(+11.4 \%)$ and CSS $(+10 \%)$, even though the overall discriminatory power of the model was only moderate. On DCA, the addition of IL6 slightly improved the clinical net benefit of the same model. Thus, preoperative IL6 and IL6sR can guide preoperative risk stratification through improved early outcome experiences the specified event whereas the grey line assumes that all patients will experience the specified event at a specific threshold probability. The dashed black line represents the net benefit of a basic reference model which was fitted using above mentioned variables. The dashed colored lines represent the net benefit of the same reference model which also includes the preoperative IL6 and IL6SR plasma levels as a variable

prognostication and may help improve patient selection for neoadjuvant systemic therapy [11-15, 24].

Both biomarkers also demonstrated the ability to improve outcome prediction if added to a model that consisted of established histopathological variables. In this postoperative setting, our prognostic model exhibited a high overall discriminatory power (C-Index of 0.79 and 0.81 for prediction of RFS and CSS, respectively). Such an accurate outcome prediction would allow for tailored therapy and thus improve patient care. However, even the addition of both biomarkers did not improve the discriminatory power for prediction of OS, suggesting that both are cancer-specific biomarkers. On DCA, however, the addition of preoperative IL6sR slightly improved the net benefit of this model for prediction of both RFS and CSS. Our findings warrant further validation of preoperative IL6 and IL6sR in the context of correlative biomarker assessment integrated in prospective clinical trials [30]. Furthermore, both carry other advantageous features of clinically beneficial biomarkers, as they are easily accessible, cost-effective and allow early outcome prediction [31]. Since IL6 and IL6sR are systemic 

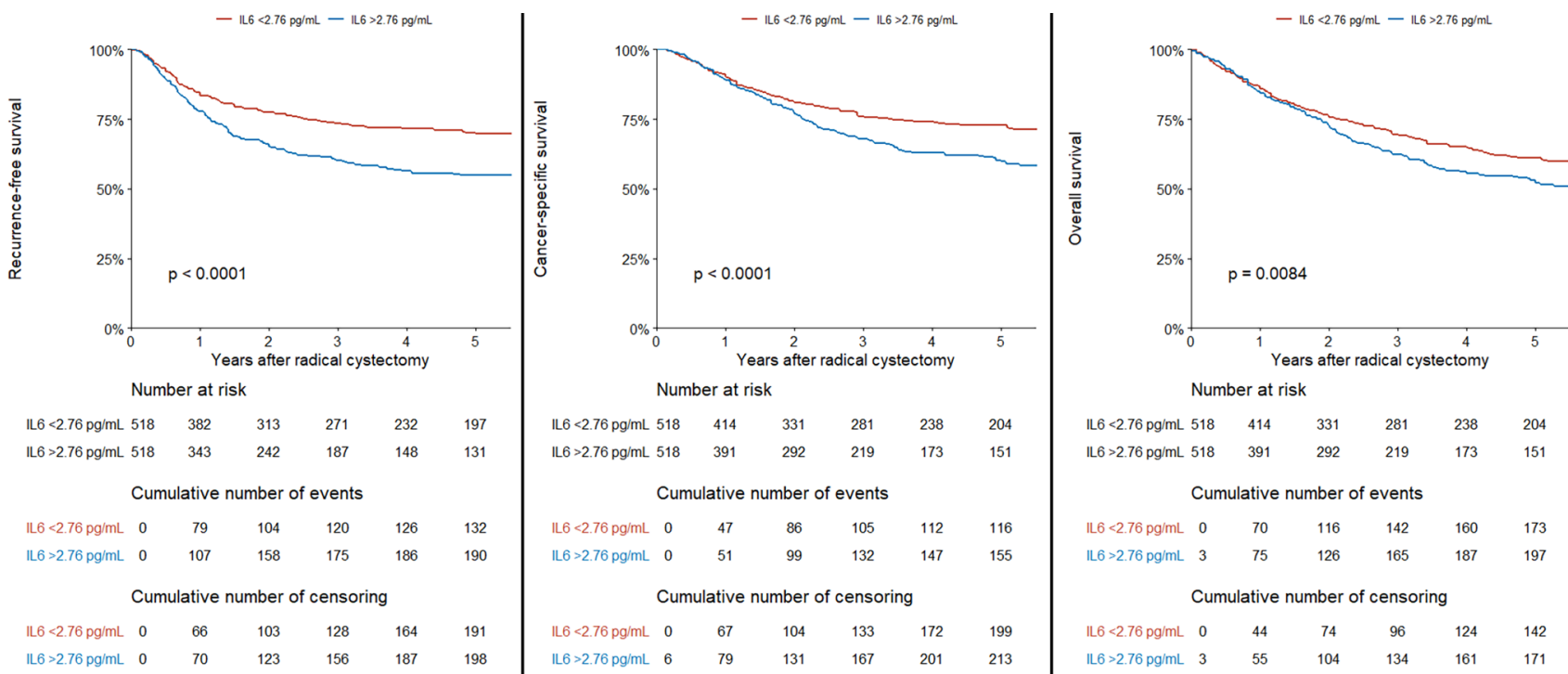

Five-year survival rates:

IL6 <2.76 pg/mL: $69.7(95 \% \mathrm{Cl} 65.4-74.3)$

IL6 >2.76 pg/mL: $54.9(95 \% \mathrm{Cl} 50.1-60.1)$

Hazard ratio: $1.69(95 \% \mathrm{Cl} 1.36-2.11) p<0.001$

Five-year survival rates:

IL6 <2.76 pg/mL: $72.8(95 \% \mathrm{Cl}$ 68.6-77.2)

IL6 >2.76 pg/mL: $59.4(95 \% \mathrm{Cl} 54.9-65.2)$

Five-year survival rates:

IL6 <2.76 pg/mLR: 61.1 (95\% Cl 56.7-66.0)

Hazard ratio: $1.63(95 \% \mathrm{Cl} 1.3-2.05) p<0.001$

IL6 >2.76 pg/mL: $52.3(95 \% \mathrm{Cl} 47.5-57.6)$

Hazard ratio: 1. $25(95 \% \mathrm{Cl} 1.06-1.47) p<0.009$

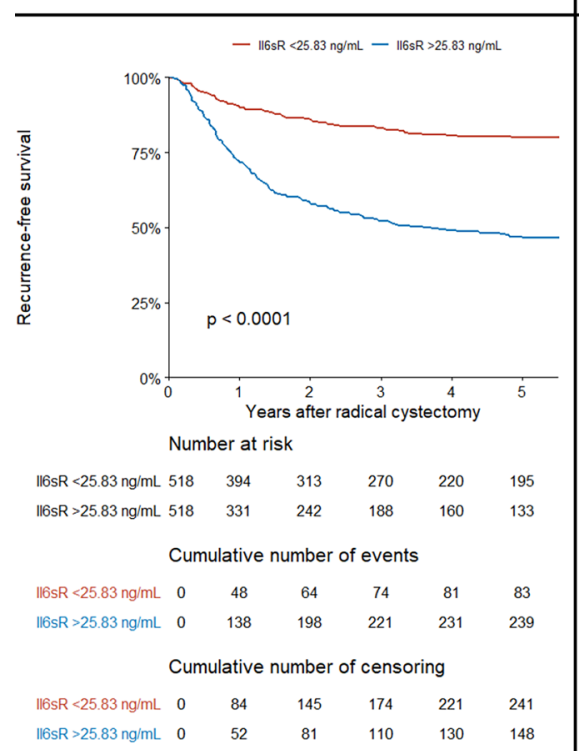

Five-year survival rates:

II6sR <25.83 ng/mL: 79.9 (95\%Cl 76.0-84.0)

II6sR > $25.83 \mathrm{ng} / \mathrm{mL}: 46.5(95 \% \mathrm{Cl} 41.9-51.6)$

Hazard ratio: $3.35(95 \% \mathrm{Cl} 2.62-4.28) p<0.001$

\section{Five-year survival rates:}

II6sR <25.83 ng/mL: 84.1 (95\% Cl 80.5-88.0)

II6sR $>25.83 \mathrm{ng} / \mathrm{mL}: 51.1(95 \% \mathrm{Cl} 46.4-56.2)$

Hazard ratio: $3.91(95 \% \mathrm{Cl} 2.98-5.12) p<0.001$

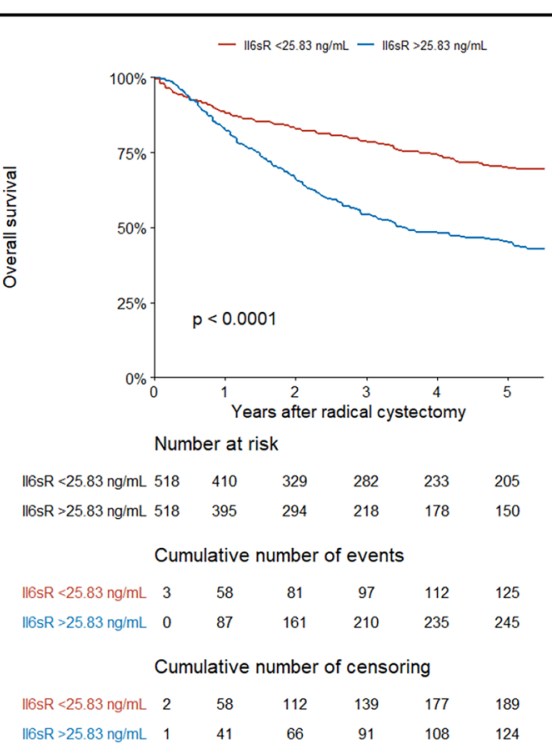

Five-year survival rates:

II6sR <25.83 ng/mL: $69.4(95 \% \mathrm{Cl} 65.4-75.6)$

Il6sR > $25.83 \mathrm{ng} / \mathrm{mL}: 45.1(95 \% \mathrm{Cl} 40.6-50.1)$

Hazard ratio: $1.8(95 \% \mathrm{Cl} 1.52-2.13) \mathrm{p}<0.001$
Fig. 2 Kaplan-Meier curves, pairwise log rank tests and five-year survival analysis with respect to recurrence-free survival (left column); cancer-specific survival (middle column) and overall survival (right column) stratified by the preoperative median interleukin-6 (i16) plasma level (first row) and the interleukin-6 soluble receptor (il6sr) plasma level (second row) in 1,036 patients treated with radical cystectomy for urothelial carcinoma of the bladder inflammatory markers, they might prove especially useful in the prediction of response to immunotherapy. Targeting IL6 and IL6sR through monoclonal antibodies is being researched as a novel treatment strategy in UCB, and in this particular setting, plasma levels of IL6 and IL6sR could permit individualization of therapy [32]. In summary, our findings warrant the inclusion of these biomarkers into future predictive/prognostic models in order to increase 


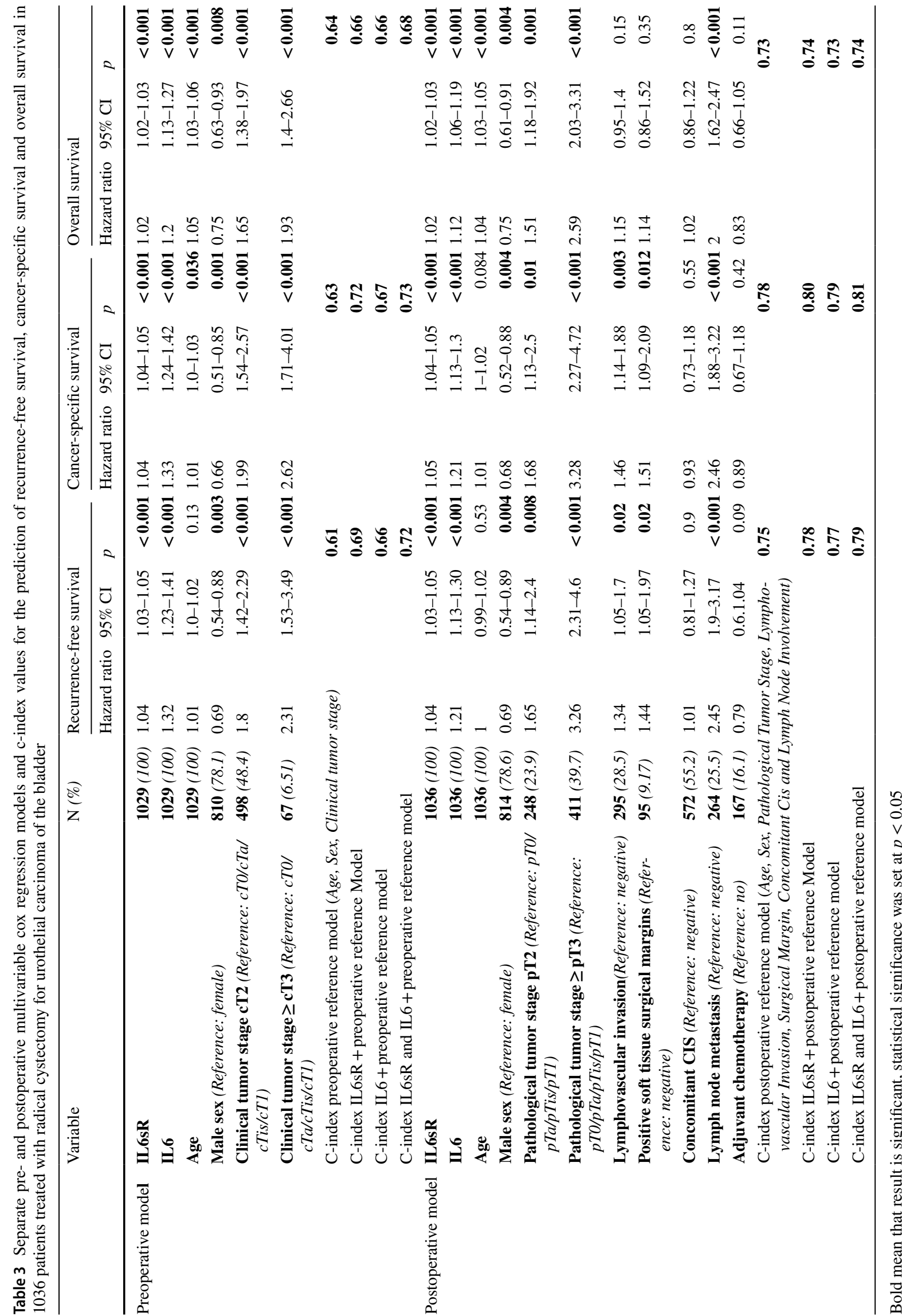


the discriminatory power and allow a personalized medicine approach, as patients with elevated biomarker levels are more likely to harbor adverse pathological features and experience poor survival outcomes.

The main limitation of our study is its retrospective analysis. Also, only the pretreatment plasma levels of IL6 and IL6sR levels were assessed in this study. Confounding conditions, such as undiagnosed infectious diseases or unknown drug interaction, could potentially have affected plasma levels of IL6 and IL6sR. However, this would have weakened an existing potential association. Data on therapies before $\mathrm{RC}$, which might also cause inflammation and alter levels of IL6 and IL6sR, such as intravesical BCG instillations, were unfortunately, unavailable. Due to the time of recruitment of this study, there is no information available on the predictive value of IL6 and IL6sR with respect to immunotherapies or neoadjuvant chemotherapy. The strength of this cohort is it purity in treatment allocation. Its weakness is that it does not reflect current treatment standards. Another limitation is that the short median follow-up with 37 months. However, we and others have shown that over two-thirds of patients experience disease recurrence after RC within 12 months and $\geq 90 \%$ in 24 months [33].

\section{Conclusions}

We externally validated that elevated preoperative plasma levels of IL6 and IL6sR levels are associated with worse survival in an independent international large cohort of patients treated with RC for UCB. Moreover, both biomarkers hold potential in identifying patients with adverse pathologic features that may benefit from intensified/multimodal therapy. They also demonstrated the ability to improve the discriminatory power of current prognostic models and thus can help guide clinical decision making.

\begin{abstract}
Authors' contribution All authors contributed to the study conception and design. Material preparation, data collection and analysis were performed by Victor Schuettfort and Shahrokh F. Shariat. The first draft of the manuscript was written by Victor Schuettfort and all authors commented on previous versions of the manuscript. All authors read and approved the final manuscript. VMS and SFS contributed to conception and design of the study, acquisition of data, analysis and interpretation of data, drafting the article. BP, DD`A, FQ, HM, EL, KM, RSM, MR, PIK, PC, JYCT, YL, DS, MA, MM and QDT contributed to analysis and interpretation of data, drafting the article and revisions.
\end{abstract}

Funding Open access funding provided by Medical University of Vienna. No funding was received for this study.

Availability of data and material The datasets generated during and/or analyzed during the current study are available from the corresponding author on reasonable request.
Code availability Throughout the manuscript, only basic and established statistical tests are applied. The codes are available on reasonable request.

\section{Declarations}

Conflicts of interest All authors have no conflict of interests to disclose.

Ethical Standards This retrospective chart review study involving human participants was in accordance with the ethical standards of the institutional and national research committee and with the 1964 Helsinki Declaration and its later amendments or comparable ethical standards. The Institutional Review Board for the Protection of Human Subjects approved this study.

Open Access This article is licensed under a Creative Commons Attribution 4.0 International License, which permits use, sharing, adaptation, distribution and reproduction in any medium or format, as long as you give appropriate credit to the original author(s) and the source, provide a link to the Creative Commons licence, and indicate if changes were made. The images or other third party material in this article are included in the article's Creative Commons licence, unless indicated otherwise in a credit line to the material. If material is not included in the article's Creative Commons licence and your intended use is not permitted by statutory regulation or exceeds the permitted use, you will need to obtain permission directly from the copyright holder. To view a copy of this licence, visit http://creativecommons.org/licenses/by/4.0/.

\section{References}

1. Witjes J, Bruins M, Cathomas R, Compérat R, Cowan N, Gakis G, et al. (2019) EAU Guidelines on muscle-invasive and metastatic bladder cancer. EAU Guidelines (2019 Edn)

2. Babjuk M, Burger M, Comperat EM, Gontero P, Mostafid AH, Palou J et al (2019) European association of urology guidelines on non-muscle-invasive bladder cancer (TaT1 and carcinoma In situ)2019 update. Eur Urol 76(5):639-657

3. Gontero P, Sylvester R, Pisano F, Joniau S, Vander Eeckt K, Serretta V et al (2015) Prognostic factors and risk groups in T1G3 non-muscle-invasive bladder cancer patients initially treated with Bacillus Calmette-Guerin: results of a retrospective multicenter study of 2451 patients. Eur Urol 67(1):74-82

4. Isbarn $\mathrm{H}$, Jeldres $\mathrm{C}$, Zini L, Perrotte P, Baillargeon-Gagne S, Capitanio U et al (2009) A population based assessment of perioperative mortality after cystectomy for bladder cancer. J Urol 182(1):70-77

5. Xylinas E, Rink M, Robinson BD, Lotan Y, Babjuk M, Brisuda A et al (2013) Impact of histological variants on oncological outcomes of patients with urothelial carcinoma of the bladder treated with radical cystectomy. Eur J Cancer 49(8):1889-1897

6. Svatek RS, Shariat SF, Lasky RE, Skinner EC, Novara G, Lerner SP et al (2010) The effectiveness of off-protocol adjuvant chemotherapy for patients with urothelial carcinoma of the urinary bladder. Clin Cancer Res 16(17):4461-4467

7. Svatek RS, Shariat SF, Novara G, Skinner EC, Fradet Y, Bastian PJ et al (2011) Discrepancy between clinical and pathological stage: external validation of the impact on prognosis in an international radical cystectomy cohort. BJU Int 107(6):898-904

8. Aziz A, May M, Burger M, Palisaar RJ, Trinh QD, Fritsche $\mathrm{HM}$ et al (2014) Prediction of 90-day mortality after radical 
cystectomy for bladder cancer in a prospective European multicenter cohort. Eur Urol 66(1):156-163

9. Rink M, Chun FK, Dahlem R, Soave A, Minner S, Hansen J et al (2012) Prognostic role and HER2 expression of circulating tumor cells in peripheral blood of patients prior to radical cystectomy: a prospective study. Eur Urol 61(4):810-817

10. Kluth LA, Black PC, Bochner BH, Catto J, Lerner SP, Stenzl A et al (2015) Prognostic and prediction tools in bladder cancer: a comprehensive review of the literature. Eur Urol 68(2):238-253

11. Soria F, Krabbe LM, Todenhofer T, Dobruch J, Mitra AP, Inman BA et al (2019) Molecular markers in bladder cancer. World J Urol 37(1):31-40

12. Novara G, Svatek RS, Karakiewicz PI, Skinner E, Ficarra V, Fradet $Y$ et al (2010) Soft tissue surgical margin status is a powerful predictor of outcomes after radical cystectomy: a multicenter study of more than 4,400 patients. J Urol 183(6):2165-2170

13. Mari A, Campi R, Tellini R, Gandaglia G, Albisinni S, Abufaraj $M$ et al (2018) Patterns and predictors of recurrence after open radical cystectomy for bladder cancer: a comprehensive review of the literature. World J Urol 36(2):157-170

14. Shariat SF, Margulis V, Lotan Y, Montorsi F, Karakiewicz PI (2008) Nomograms for bladder cancer. Eur Urol 54(1):41-53

15. Shariat SF, Youssef RF, Gupta A, Chade DC, Karakiewicz PI, Isbarn $\mathrm{H}$ et al (2010) Association of angiogenesis related markers with bladder cancer outcomes and other molecular markers. J Urol 183(5):1744-1750

16. Shariat SF, Chade DC, Karakiewicz PI, Ashfaq R, Isbarn H, Fradet $Y$ et al (2010) Combination of multiple molecular markers can improve prognostication in patients with locally advanced and lymph node positive bladder cancer. J Urol 183(1):68-75

17. Shariat SF, Lotan Y, Vickers A, Karakiewicz PI, Schmitz-Drager BJ, Goebell PJ et al (2010) Statistical consideration for clinical biomarker research in bladder cancer. Urol Oncol 28(4):389-400

18. Zhu Z, Shen Z, Xu C (2012) Inflammatory pathways as promising targets to increase chemotherapy response in bladder cancer. Mediators Inflamm 2012:528690

19. Chen MF, Lin PY, Wu CF, Chen WC, Wu CT (2013) IL-6 expression regulates tumorigenicity and correlates with prognosis in bladder cancer. PLoS ONE 8(4):e61901

20. Okamoto M, Hattori K, Oyasu R (1997) Interleukin-6 functions as an autocrine growth factor in human bladder carcinoma cell lines in vitro. Int J Cancer 72(1):149-154

21. Shariat SF, Kattan MW, Traxel E, Andrews B, Zhu K, Wheeler $\mathrm{TM}$ et al (2004) Association of pre-and postoperative plasma levels of transforming growth factor beta(1) and interleukin 6 and its soluble receptor with prostate cancer progression. Clin Cancer Res 10(6):1992-1999

22. Taher MY, Davies DM, Maher J (2018) The role of the interleukin (IL)-6/IL-6 receptor axis in cancer. Biochem Soc Trans 46(6):1449-1462
23. Andrews B, Shariat SF, Kim JH, Wheeler TM, Slawin KM, Lerner SP (2002) Preoperative plasma levels of interleukin- 6 and its soluble receptor predict disease recurrence and survival of patients with bladder cancer. J Urol 167(3):1475-1481

24. Moschini M, Soria F, Klatte T, Wirth GJ, Özsoy M, Gust K et al (2017) Validation of preoperative risk grouping of the selection of patients most likely to benefit from neoadjuvant chemotherapy before radical cystectomy. Clin Genitourin Cancer 15(2):e267-e273

25. Shariat SF, Andrews B, Kattan MW, Kim J, Wheeler TM, Slawin KM (2001) Plasma levels of interleukin-6 and its soluble receptor are associated with prostate cancer progression and metastasis. Urology 58(6): 1008-1015

26. Shariat SF, Kim J, Raptidis G, Ayala GE, Lerner SP (2003) Association of p53 and p21 expression with clinical outcome in patients with carcinoma in situ of the urinary bladder. Urology 61(6):1140-1145

27. Shariat SF, Khoddami SM, Saboorian H, Koeneman KS, Sagalowsky AI, Cadeddu JA et al (2004) Lymphovascular invasion is a pathological feature of biologically aggressive disease in patients treated with radical prostatectomy. J Urol 171(3):1122-1127

28. Rink M, Fajkovic H, Cha EK, Gupta A, Karakiewicz PI, Chun FK et al (2012) Death certificates are valid for the determination of cause of death in patients with upper and lower tract urothelial carcinoma. Eur Urol 61(4):854-855

29. D'Andrea D, Soria F, Zehetmayer S, Gust KM, Korn S, Witjes JA et al (2019) Diagnostic accuracy, clinical utility and influence on decision-making of a methylation urine biomarker test in the surveillance of non-muscle-invasive bladder cancer. BJU Int 123(6):959-967

30. Lotan Y, Shariat SF, Schmitz-Drager BJ, Sanchez-Carbayo M, Jankevicius F, Racioppi M et al (2010) Considerations on implementing diagnostic markers into clinical decision making in bladder cancer. Urol Oncol 28(4):441-448

31. Bensalah K, Montorsi F, Shariat SF (2007) Challenges of cancer biomarker profiling. Eur Urol 52(6):1601-1609

32. Miyake M, Furuya H, Onishi S, Hokutan K, Anai S, Chan O et al (2019) Monoclonal Antibody against CXCL1 (HL2401) as a novel agent in suppressing il6 expression and tumoral growth. Theranostics 9(3):853-867

33. Rink M, Lee DJ, Kent M, Xylinas E, Fritsche HM, Babjuk M et al (2013) Predictors of cancer-specific mortality after disease recurrence following radical cystectomy. BJU Int 111(3b):E30-6

Publisher's Note Springer Nature remains neutral with regard to jurisdictional claims in published maps and institutional affiliations.

\title{
Authors and Affiliations
}

\author{
Victor M. Schuettfort ${ }^{1,2}\left(\right.$ D $\cdot$ Benjamin Pradere ${ }^{1} \cdot$ Quoc-Dien Trinh $^{3} \cdot$ David D'Andrea $^{1} \cdot$ Fahad Quhal $^{1,4}$. \\ Hadi Mostafaei ${ }^{1,5}$. Ekaterina Laukhtina ${ }^{1,6} \cdot$ Keiichiro Mori ${ }^{1,7} \cdot$ Reza Sari Motlagh $^{1} \cdot$ Michael Rink $^{2}$. \\ Pierre I. Karakiewicz ${ }^{8}$. Piotr Chlosta ${ }^{9}$. Jeremy Yuen-Chun Teoh ${ }^{10}$. Yair Lotan ${ }^{11}$. Douglas Scherr ${ }^{12}$. \\ Mohammad Abufaraj ${ }^{1,13}$. Marco Moschini ${ }^{14,15,16}$. Shahrokh F. Shariat ${ }^{1,6,11,12,13,17,18,19}$
}

1 Department of Urology, Comprehensive Cancer Center, Vienna General Hospital, Medical University of Vienna, Währinger Gürtel 18-20, 1090 Vienna, Austria
2 Department of Urology, University Medical Center Hamburg-Eppendorf, Hamburg, Germany 
3 Division of Urological Surgery and Center for Surgery and Public Health, Brigham and Women's Hospital, Harvard Medical School, Boston, MA, USA

4 Department of Urology, King Fahad Specialist Hospital, Dammam, Saudi Arabia

5 Research Center for Evidence Based Medicine, Tabriz University of Medical Sciences, Tabriz, Iran

6 Institute for Urology and Reproductive Health, Sechenov University, Moscow, Russia

7 Department of Urology, The Jikei University School of Medicine, Tokyo, Japan

8 Cancer Prognostics and Health Outcomes Unit, Division of Urology, University of Montreal Health Center, Montreal, Canada

9 Department of Urology, Medical College, Jagiellonian University, Krakow, Poland

10 S.H. Ho Urology Centre, Department of Surgery, Prince of Wales Hospital, The Chinese University of Hong Kong, Hong Kong, China

11 Department of Urology, University of Texas Southwestern, Dallas, TX, USA
12 Department of Urology, Weill Cornell Medical College, New York Presbyterian Hospital, New York, USA

13 Division of Urology, Department of Special Surgery, Jordan University Hospital, The University of Jordan, Amman, Jordan

14 Department of Urology, Luzerner Kantonsspital, Lucerne, Switzerland

15 Department of Urology, Institut Mutualiste Montsouris, Paris, France

16 Department of Urology and Division of Experimental Oncology, Urological Research Institute, Vita-Salute San Raffaele, Milan, Italy

17 Department of Urology, Hospital Motol, Second Faculty of Medicine, Charles University, Praha, Czech Republic

18 Karl Landsteiner Institute of Urology and Andrology, Vienna, Austria

19 European Association of Urology Research Foundation, Arnhem, Netherlands 